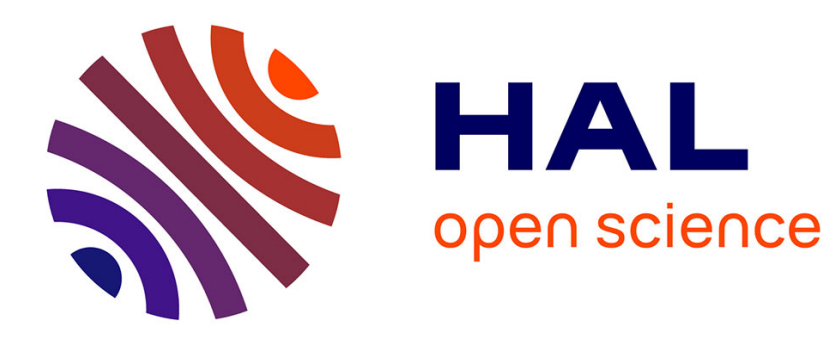

\title{
Techniques de plantation des feuillus précieux: caractéristiques des plants de Merisier, Frêne et Érable sycomore
}

Gérard Armand

\section{- To cite this version:}

Gérard Armand. Techniques de plantation des feuillus précieux: caractéristiques des plants de Merisier, Frêne et Érable sycomore. Revue forestière française, 1992, 44 (S), pp.66-70. $10.4267 / 2042 / 26384$. hal-03443874

\section{HAL Id: hal-03443874 \\ https://hal.science/hal-03443874}

Submitted on 23 Nov 2021

HAL is a multi-disciplinary open access archive for the deposit and dissemination of scientific research documents, whether they are published or not. The documents may come from teaching and research institutions in France or abroad, or from public or private research centers.
L'archive ouverte pluridisciplinaire HAL, est destinée au dépôt et à la diffusion de documents scientifiques de niveau recherche, publiés ou non, émanant des établissements d'enseignement et de recherche français ou étrangers, des laboratoires publics ou privés. 


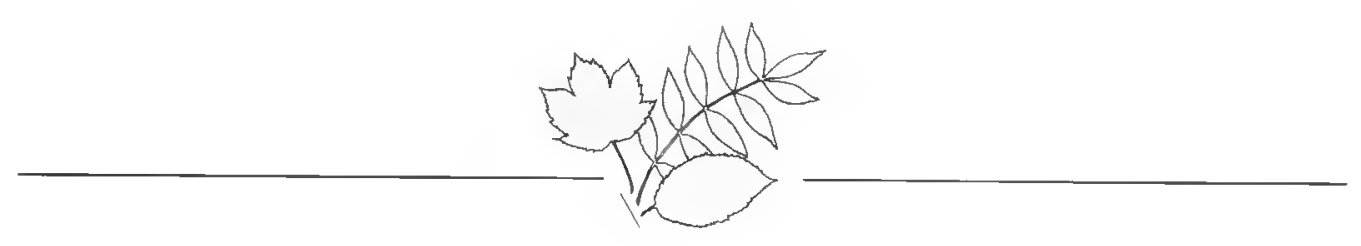

\section{TECHNIQUES DE PLANTATION DES FEUILLUS PRÉCIEUX: CARACTÉRISTIQUES DES PLANTS DE MERISIER, FRÊNE ET ÉRABLE SYCOMORE}

\section{G. ARMAND}

L'objectif d'une production rapide, au moindre coût, d'un maximum de bois de qualité à partir des plantations de feuillus précieux ne sera atteint que si on installe au départ un matériel végétal performant et adapté.

Le matériel végétal se caractérise, outre son origine, par des critères de qualité bien définis.

\section{HISTORIQUE}

Les premières installations importantes de ces espèces par des forestiers privés de l'Est de la France datent de 1974. Les plants utilisés avaient une taille de 50 à $80 \mathrm{~cm}$. Ils étaient d'origine inconnue, âgés de 1 ou 2 ans, avec très souvent un mauvais équilibre racine-tige.

Face à cette situation, les organismes de la forêt privée de l'Est ont souhaité maîtriser eux-mêmes la production des plants en créant, en 1976, un réseau de pépinières chez les sylviculteurs.

Les bons résultats de cette expérience ont permis de mettre à la disposition des reboiseurs des plants d'origine connue, de bonne qualité. Ces pépinières ont notamment produit des grands plants destinés aux plantations, installées à faible densité ( 400 à 600 plants/ha) pour réduire les coûts, qu'on devrait protéger des dégâts croissants de cervidés.

À partir de 1982, des essais ont porté sur des types de plants élevés en culture accélérée sous tunnel, en conteneurs ou non.

\section{BILAN APRĖS 17 ANNÉES D'EXPÉRIENCE}

\section{Qu'est-ce qu'un bon plant?}

C'est un plant:

- de bonne origine et suffisamment "plastique", 
— à tige droite bien lignifiée sans branches latérales,

— de dimensions maximales (hauteur, volume de racines, grosseurs du bourgeon) pour un âge jamais supérieur à 3 ans,

- d'indice d'élancement (hauteur/diamètre au collet) compris entre 50 et 70 ,

- dont l'enracinement aura été maintenu frais jusqu'à sa mise en terre (par l'usage d'enveloppes par exemple).

Types de plants proposés, avantages, inconvénients

Les types de plants sont au nombre de deux:

- petits plants élevés en pleine terre:

- 60-80 cm en 1 an $(1+0)$ pour Érable et Merisier,

$-25-50 \mathrm{~cm}$ en 1 an $(1+0)$ pour le Frêne.

Ces plants sont peu onéreux à la mise en terre, ils corrigent mieux leurs défauts de forme dus à un départ difficile. Ils sont à recommander pour les boisements sur anciennes terres agricoles à densitè supérieure à 400/ha.

Par contre, ils peuvent entraîner un surcoût dû à leur taille, et aux dégagements en forêt et éventuellement en protection.

- grands plants élevés en pleine terre:

- 125-175 cm en 2 ans $(1+1)$ pour Merisier,

- 125-175 cm en 2 ans $(1+1)$ pour Érable,

$-125-175 \mathrm{~cm}$ en 3 ans $(1+2)$ pour Frêne.

Ils coûtent le double des petits plants à l'achat et à la mise en terre. Le surcoût n'est pas compensé par l'économie faite sur les entretiens aux sols et aux arbres. En cas de mauvaise reprise, les déformations situées à la hauteur initiale sont difficilement rectifiables.

A proscrire sur terre agricole, ils sont à résenver aux meilleurs sols, en présence de recrû ligneux et doivent être plantés à densité inférieure à 400 (200 à 300 à l'ha).

Sans les écarter totalement, on accorde moins d'intérêt aux plants de $100 \mathrm{~cm}$ qui ne peuvent être protégés par un manchon, et qui rentabilisent moins une protection type mini-serre ou gaine " transex " qui permet l'effet de serre.

Ce type de plant de $100 \mathrm{~cm}$ et plus représente la plus forte dimension financée par le FFN. Cette limite peut être critiquée.

De même, les très grands plants de $2 \mathrm{~m}$ et plus, très onéreux à installer, ne séduisent pas, même si, avec le Frêne, on observe dans un dispositif des Vosges des résultats très encourageants.

Les plants en conteneurs ne sont pas vraiment satisfaisants. Ils sont chers à l'achat et à l'installation. Ils ont parfois une meilleure reprise, mais ne croissent pas plus vite ensuite. Avantage néanmoins: on peut les planter feuillés en fin de saison normale.

Comparaison de la croissance entre grands plants et petits plants: exemples de deux dispositifs installés en Lorraine

- Cas du Merisier (figure 1, p. 68)

Malgré la croissance spectaculaire des petits plants $1+0$ en première saison grâce à l'effet des abris, l'avantage des plants $1+1$ s'affirme en troisième saison de végétation, pour ce qui est de la hauteur totale, en sol brun sur limon profond et sous ambiance forestière. 

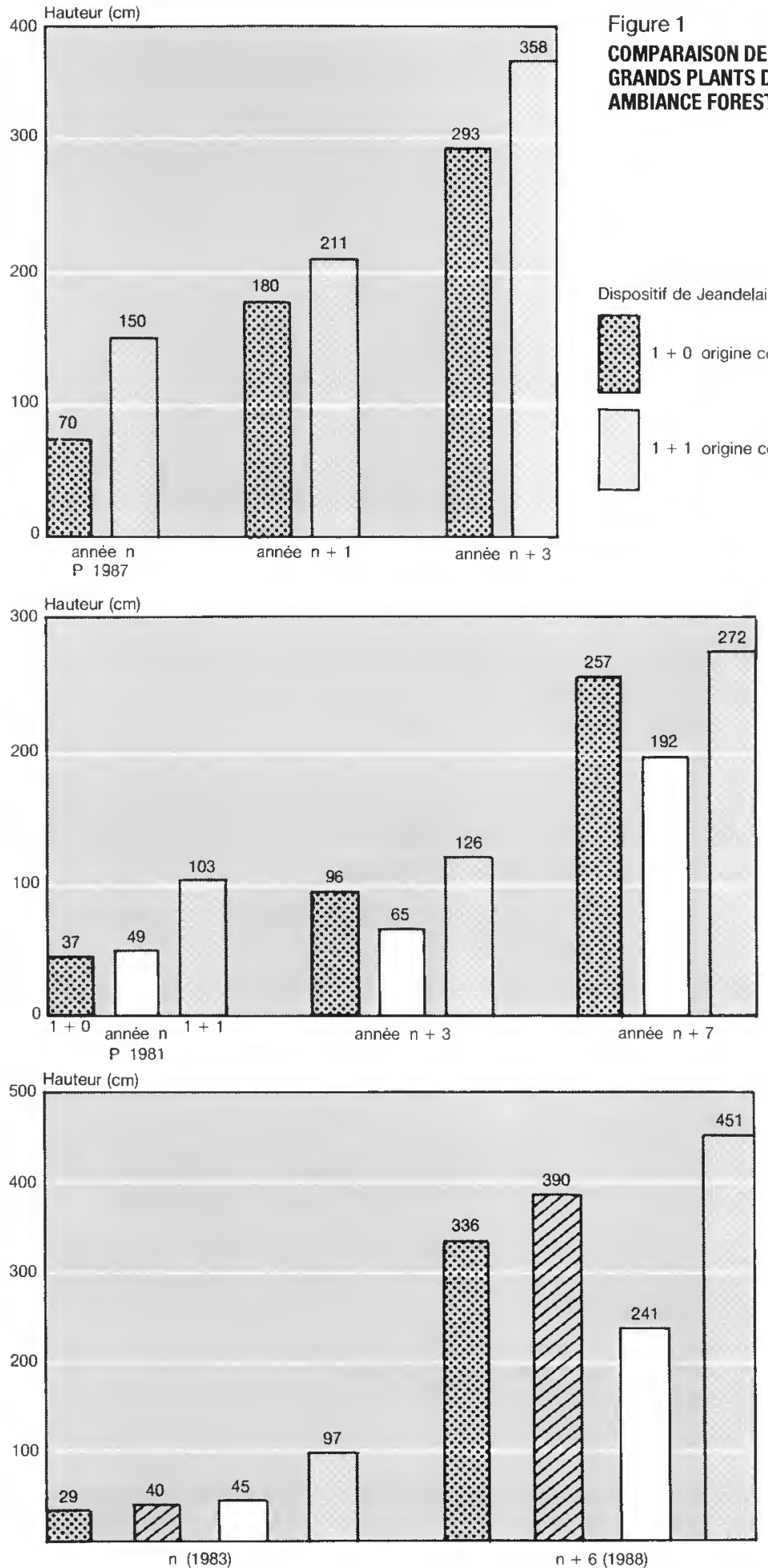

Figure 1

\section{COMPARAISON DE CROISSANCE DE PETITS ET GRANDS PLANTS DE MERISIER EN SOL BRUN ET EN AMBIANCE FORESTIĖRE}

Dispositif de Jeandelaincourt (54)

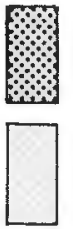

$1+0$ origine connue avec mini-serre

+1 origine connue

Figure 2

COMPARAISON DE CROISSANCE DE PETITS ET GRANDS PLANTS DE FREENE * HORS FORÉT »SUR SOL Ȧ HYDROMORPHIE TEMPORAIRE

Dispositif de Nettancourt (55)

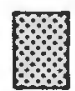

$1+0$ origine connue

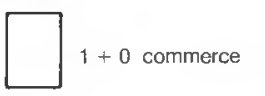

Figure 3

EXPÉRIMENTATION DE TYPES DE PLANTS D'ÉRABLE EN CONTENEURS

Dispositif de Charency-Vezin (54)

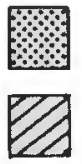
walahick Sylvagrest walahick Écorces + Tourbe

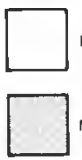
melfert racines nues 
- Cas du Frêne (figure 2, p. 68)

En sol à hydromorphie temporaire et hors forêt, en plants $1+0$, la meilleure origine locale $(67$, IIlwald) rejoint l'origine du commerce en grands plants $1+1$ après 7 saisons de végétation.

Dans ce même dispositif, les grands plants de Merisier et d'Érable sycomore ont conduit à un échec.

Ces observations sont confirmées dans d'autres parcelles d'expérimentation de l'inter-région Est.

\section{Un exemple d'essai de types de plants d'Érable en conteneur}

Dans le dispositif de Charency-Vezin (Meurthe-et-Moselle) (figure 3, p. 68), les plants à racines nues conservent leur avantage de départ après 6 saisons de végétation. Les plants en motte "Melfert " perdent peu à peu du terrain. L'ensemble est installé sur une ancienne culture en sol brun calcique et environnement forestier.

\section{Comparaison des coûts d'installation et d'entretien \\ d'un grand plant et d'un petit plant sur les postes où ils diffèrent}

Tableau I

Comparaison des coûts d'installation et d'entretien petit plant/grand plant sur les postes où ils diffèrent

Conditions: plantation en ambiance forestière contrôlée

Densité : $400 /$ ha $(7 \times 3,5 \mathrm{~m})$

Prix : - plants : base FFN (+15\% pour grand plant)

- travaux: base régie $63 \mathrm{~F} / \mathrm{h} \mathrm{HT} 1991$ (-30\% sur tarif mayen entreprise) ramenés au plant installé

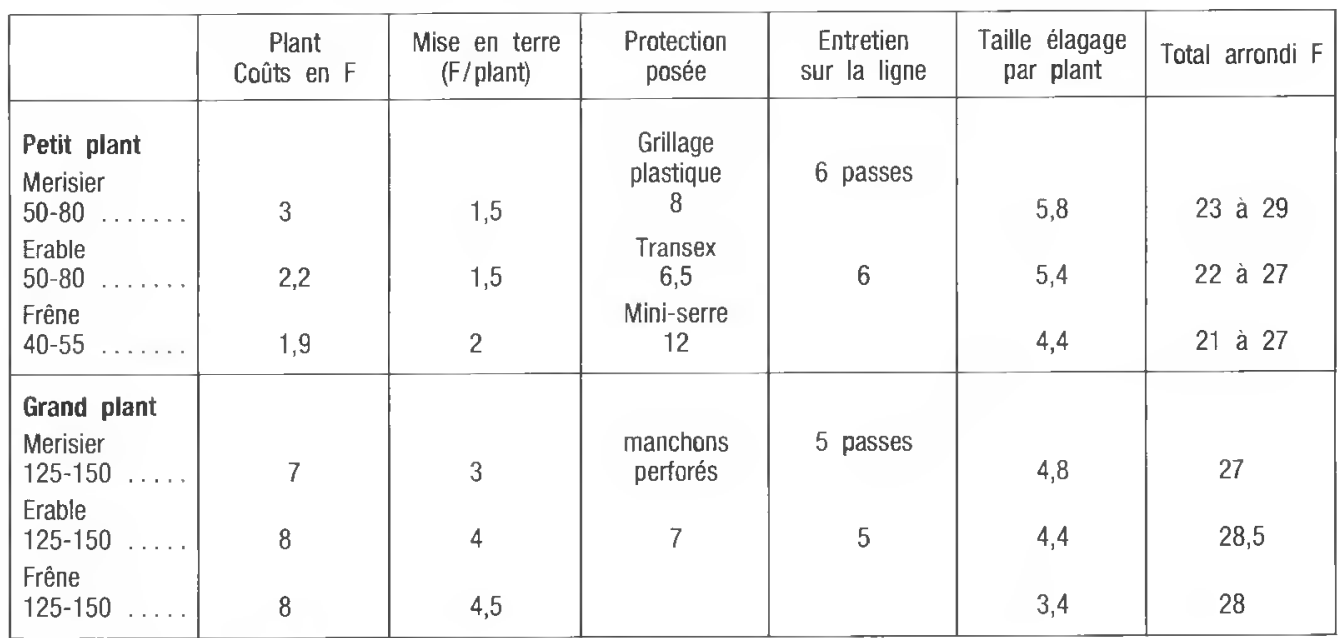

L'économie sur les entretiens pour les grands plants de Frêne et d'Erable demeure interrieure au surcoût d'installation, dont ils sont responsables au départ.

En plantation sous ambiance forestière contrôlée, à la densité de 400/ha, l'avantage global revient aux petits plants: quand on compare les coûts grand plant avec manchon et petit plant avec mini-serre, l'économie réalisée sur les entretiens pour les grands plants demeure inférieure à leur surcoût d'installation.

Seul le Merisier fait exception à ce classement.

Le petit plant devient vraiment avantageux économiquement si on le protège avec gaines Transex. 


\section{G. ARMAND}

\section{CONCLUSIONS}

Le choix de plants performants et bien adaptés est une étape importante dans la réussite d'un boisement ou reboisement. Mais la réussite du projet ne sera totale que si on les installe dans les meilleures conditions: sol propre en forêt, propre et labouré hors forêt, et si on maitrise bien par la suite les entretiens du sol et les soins aux arbres.

Alors, grand plant ou petit plant?

C'est le coût de l'opération, dans l'un et l'autre cas, qui dictera le choix, compte tenu de l'option densité forte ou faible choisie, et de la décision d'éduquer ou non les plants un à un.

\section{G. ARMAND}

FÉDÉRATION DE LA VULGARISATION FORESTIĖRE DE L'EST

11 , rue de la Commanderie

54000 NANCY 